\title{
TRANSIENT PHENOMENA IN THE ENERGETIC BEHIND-THE-LIMB SOLAR FLARE OF SEPTEMBER 29, 1989
}

\author{
A. BHATNAGAR and R.M. JAIN \\ Udaipur Solar Observatory, Udaipur, 311001, India \\ J.T. BURKEPILE \\ HAO, Boulder, Colorado, 80307, USA \\ I.M. CHERTOK \\ IZMIRAN, Troitsk, Moscow Region, 142092, Russia \\ A. MAGUN \\ IAP, University of Bern, CH-3012 Bern, Switzerland \\ H. URBARZ \\ Weissenau Station of the Astronomical Institute, \\ University of Tubingen, 7980 Ravensburg, Germany \\ and \\ P. ZLOBEC \\ Trieste Astronomical Observatory, 34131, Trieste, Italy
}

\begin{abstract}
The powerful cosmic ray flare of Sept. 29, 1989 occurred behind the limb and was observed over a wide spectral range. The analysis of optical, radio, and other relevant data suggest two phases of energy release. After an impulsive phase a prolonged post eruption energy release occurred in an extended region of the corona following the eruption of a large coronal mass ejection (CME). This phase is responsible for numerous coronal and interplanetary phenomena including the ground-level increase of cosmic rays.
\end{abstract}

Key words: Flare - CME - Post-Eruption Energy Release

\section{Introduction}

The September 29, 1989 flare is well known and studied mainly because it is one of the most important events in the list of the largest solar cosmic ray ground-level increases (see Torsti et al., 1992; Cliver et al., 1993; Vestrand and Forrest, 1993). In spite of it's occurrence behind the limb (AR 5698, S24 W100-105) the flare could be observed in intense emission over a wide range of the spectrum, from gamma-rays to decametric wavelengths.

The goal of the present contribution is the study of this flare on the basis of available optical, radio, and other relevant data. We used in particular observations made with the Razdow full disc $\mathrm{H}$-alpha monitoring telescope of the Udaipur Solar Observatory, the white-light coronagraph/polarimeter aboard the SMM satellite, the fixed-frequency polarimeters/radiometers $(169 \mathrm{MHz}-50 \mathrm{GHz})$ and radio spectrographs $(30-1000 \mathrm{MHz})$ of the Bern, IZMIRAN, Trieste, and Weissenau observatories. 

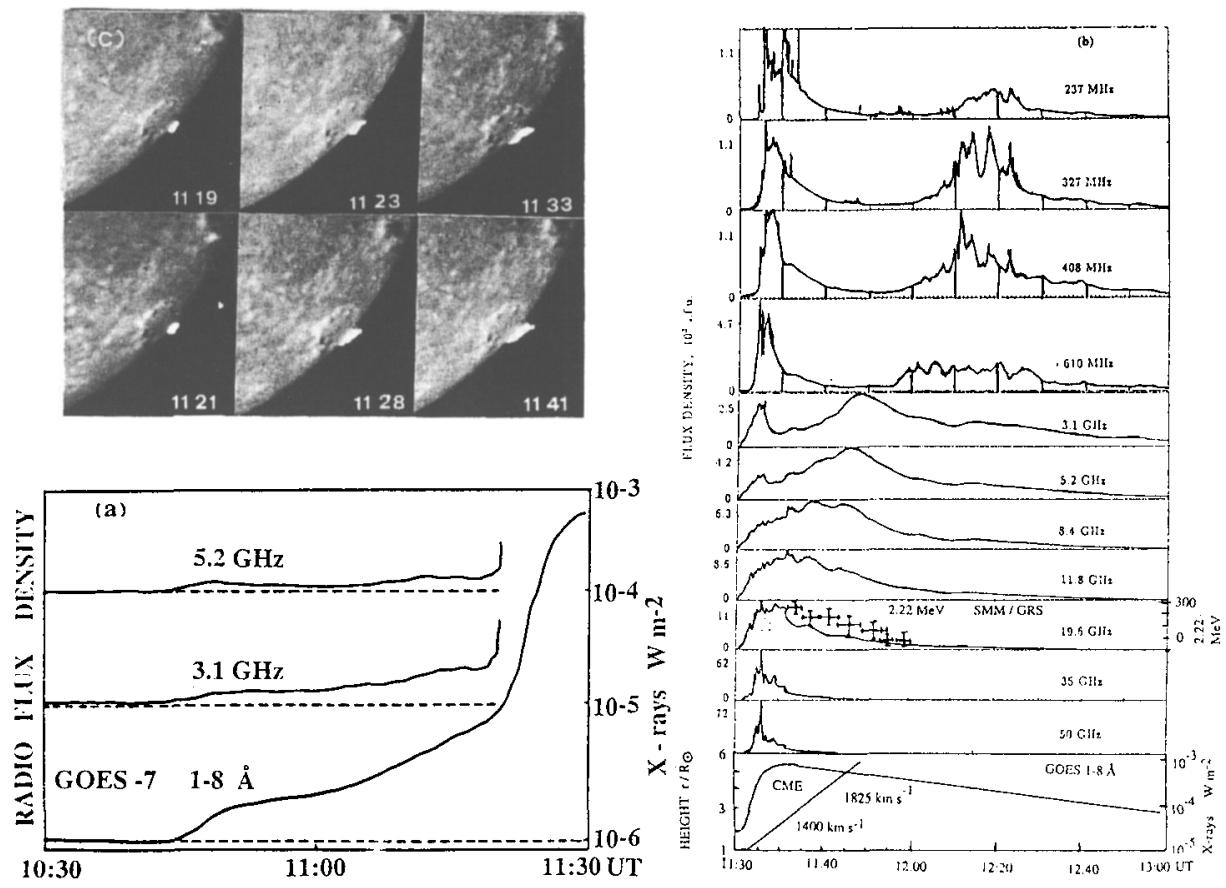

Fig. 1. Temporal development of the September 29, 1989 event. (a) The pre-burst rise in microwaves and soft X-rays. (b) The temporal evolution of the radio (Bern, Trieste) and soft X-ray (GOES-7) emission; the data points placed near the $19.6 \mathrm{GHz}$ time profile represent the 2.22 gamma-ray line intensity (Vestrand and Forrest, 1993); the lower panel shows the high-time trajectories of the CME (crosses) and the shock wave obtained from type II observations (solid points). (c) Post-flare $\mathrm{H} \alpha$ loops observed in Udaipur.

\section{Observations}

The large $(\Delta \Theta \sim 77 \mathrm{deg})$, high-speed $(V \sim 1825-2000 \mathrm{~km} / \mathrm{s})$, and massive $\left(m \sim(1-2) \times 10^{16} \mathrm{~g}\right) \mathrm{CME}$ was observed on the west limb as a bright, structured and extending loop with apparent location from about 220 to 300 degrees position angle. The extrapolated trajectory of the CME leads to a start time of the mass ejection from the solar limb at 11:22 UT which is close to the onset of the strong microwave burst and sharp increase of the soft X-ray emission (Figure 1b). However, the CME trajectory does not take into account the initial acceleration so that the real start time of the CME may be some what earlier. The $\mathrm{H} \alpha$ observations show that the CME was preceded by several eruptions above the same active region. Directly before the start of the CME under consideration, a strong eruption took place visible up to a height of about $124 \times 10^{3} \mathrm{~km}$ at 10:22 UT. In the microwave 
and soft $\mathrm{X}$-ray regions, a distinct gradual pre-burst rise took place starting from around 10:30-10:40 UT (Figure 1a).

The radio data reveal that at least two phases of the energy release occurred during this flare (Figure $1 \mathrm{~b}$ ). The first component started between 11:20 and 11:23 UT and reached its peak intensity around 11:25-11:26 UT, almost simultaneously at all frequencies between $50 \mathrm{GHz}$ to $237 \mathrm{MHz}$. The more prolonged second component became visible in microwaves below 19.6 $\mathrm{GHz}$ after 11:26 UT and later in the decimeter range. Its main feature is a clear delay of emission towards lower frequencies. Note that at the peak time of the second component, the CME front had already reached a height of about $4 R_{\odot}$ above the limb. In the dynamic spectrum at metric and decimetric wavelenghts, the emission was observed as a complicated combination of type II, III/U, and IV bursts. A conversion of the frequency drift of the starting band of the type II burst, with the use of two times the Newkirk plasma density model, shows that there was a shock driven by the CME loop. During the late stage of the event, simultaneously with a long duration soft X-ray burst, an outstanding and slowly rising post-flare $\mathrm{H} \alpha$ loop system developed (Figure 1c) and was visible for at atleast 12 hours.

\section{Discussion and Conclusion}

The data outlined above allow us to suggest the following general scenario emphasizing the role of the CME and post-eruption energy release. The CME erupts either as a consequence of an instability of the large scale coronal structures or under the influence of the primary energy release. The latter manifests itself by the first component observed at microwaves and lower frequencies. Propagating through the corona, the large CME strongly disturbs the coronal magnetic field in an extended region. After the passage of the $\mathrm{CME}$, the disturbed magnetic field relaxes to its initial state via magnetic field reconnection in a quasi vertical current sheet. This is accompanied by the prolonged post-eruption energy release and particle acceleration in the corona, well after the impulsive phase (e.g. Švestka, 1989). The restructuring of the coronal magnetic field leads to the forming of higher and higher loops. In the beginning these hot loops give rise to soft X-ray emission and after cooling down become observable in $\mathrm{H} \alpha$. This post-eruption energy release appears to be mainly responsible for the observed second or delayed burst component.

Moreover, the recent analysis of high-energy, gamma-ray and neutron events of long duration provide strong evidence. For example, in large events, the post-eruption energy release results in a long term particle acceleration to high energies, in particular of protons up to several $\mathrm{GeV}$ at the later stages of the flare (see Akimov et al., 1995; Chertok, 1995). 
The post eruption-energy release can create spatially extended sources of broad band emission covering large areas on the solar disk. In particular, the observability of the gamma-ray event (Vestrand and Forrest, 1993) may be due to particle precipitation through large scale magnetic field loops that connect the source of the post-eruption energy release above the active region behind the limb (AR 5698) with the region (AR 5703) located on the disk (S24 W62). This interpretation seems to be more likely than suggestion that a CME-driven coronal shock is a plausible source of the energetic protons producing gamma-rays on the visible disk (Cliver et al., 1993).

We also suggested that the post-eruption particle acceleration can be responsible for the long duration increase of the low-energy (tens of $\mathrm{MeV}$ ) proton fluxes in the interplanetary space. An important additional effect here is that the particles accelerated in a process of the post-eruption energy release may be trapped inside the propagating CME and then leak out gradually into the interplanetary space.

According to Torsti et al. (1992) the ground-level high-energy cosmic ray increase, associated with the Sept. 29, 1989 flare, also seems to reveal two subsequent particle ejections from the Sun with a time difference of several tens of minutes. Our observational results of two phases, allow us to associate these with the initial and post-eruption particle accelerations respectively.

Thus, the September 29, 1989 flare is one more outstanding example of events in which the CME and the related post-eruption energy release play a significant role both in solar coronal processes and their interplanetary consequences including cosmic rays.

\section{Acknowledgements}

I.M.Ch. acknowledges support from the Russian Foundation of Fundamental Researches and State Astronomical Program, the International Science Foundation, and the ESO C\&EE Program. We also thank the Swiss National Science Foundation, grant 20-36417.92, for financial support. Chromospheric observations were made at the Udaipur Solar Observatory under an Indo-US project.

\section{References}

Akimov, A.A., Ambrož, P., Belov, A.V., et al.: 1995, Preprint of IAP, Bern, 44 p.; Solar Phys. (in press).

Chertok, I.M.: 1995, in T.Watanabe, ed, Proc. of the Second SOLTIP Symp., Ibaraki University, Mito, (in press).

Cliver, E.W., Kahler, S.W., and Vestrand, W.T.: 1993, 23rd ICRC, Calgary, 3, 91.

Švestka, Z.: 1989, Solar Phys. 121, 399. 
Torsti, J.J., Eronen, T., Mähönen M., et al.: 1992, in E.Marsch and R.Schwenn, eds., Solar Wind Seven, Pergamon Press, Oxford, 545.

Vestrand, W.T. and Forrest, D.J.: 1993, Astrophys. J. (Letters), 409, L69. 